Turkmani S, Homer CSE, Dawson A. Maternity care experiences and health needs of migrant women from female genital mutilation-practicing countries in high-income contexts: A systematic review and meta-synthesis, Birth, 2019, 46 (1), pp. 3 - 14, http://hdl.handle.net/10453/135578

\title{
Maternity care experiences and health needs of migrant women from female genital mutilation practising countries in high-income contexts: A systematic review and meta-synthesis
}

\begin{abstract}
Background: Female genital mutilation (FGM) is a cultural practice defined as the partial or total removal of the external female genitalia for non-therapeutic indications. Due to changing patterns of migration, clinicians in high-income countries are seeing more women from countries where the practice is prevalent. This review aimed to understand the socio-cultural and health needs of these women and identify opportunities to improve the quality of maternity care for women with FGM.
\end{abstract}

Methods: We undertook a systematic review and meta-synthesis of peer reviewed primary qualitative research to explore the experience and needs of migrant women with FGM receiving maternity care. A structured search of nine databases was undertaken, screened papers appraised and a thematic analysis undertaken on data extracted from the findings and discussion sections of included papers.

Results: Sixteen peer reviewed studies were included in the systematic review. Four major themes were revealed: Living with fear, stigma and anxiety; Feelings of vulnerability, distrust and discrimination; Dealing with past and present ways of life after resettlement, and; Seeking support and involvement in health care.

Conclusions: The findings suggest that future actions for improving maternity care quality should be focused on woman-centred practice, demonstrating cultural safety and developing mutual trust between 
a woman and her care providers. Meaningful consultation with women affected by FGM in high-income settings requires cultural sensitivity and acknowledgement of their specific circumstances. This can be achieved by engaging women affected by FGM in service design to provide quality care and ensure woman focused policy is developed and implemented.

Keywords: Female genital mutilation (FGM), maternity care, high-income countries. 


\section{Background}

Female genital mutilation (FGM) is defined as partial or total removal of external female genitalia for non-therapeutic indications. ${ }^{1}$ This practice is deeply rooted in culture, with social obligation, rites of passage, marriageability, the maintenance of marital fidelity and aesthetics considered to be the primary reasons for its continuation. ${ }^{1,2}$

There are more than 200 million women and girls with FGM concentrated in 29 countries in Africa, the Middle East and Asia. ${ }^{1,3}$ Globally, the prevalence of FGM is declining and the proportion of girls being cut is lower than those from their mothers' generation. ${ }^{4}$ However, increasing population growth and migration means that the total number of girls subjected to this practice is likely to grow and extend to high-income countries where FGM is not traditionally practiced. ${ }^{1,5,6}$, In Belgium for example, the estimated proportion of women affected by FGM is $2.7 \%^{7}$ and in London $2.1 \%{ }^{8}$

FGM is associated with higher rates of obstetric interventions and complications during labour and birth. ${ }^{9,10}$ Migrant women from FGM prevalent countries have been found to be at risk of a higher incidence of adverse pregnancy outcomes. For example, a meta-analysis of studies from six high-income countries showed that migrant women from Somalia have higher rates of caesarean section and stillbirth than local women and experience issues accessing maternity services. ${ }^{11}$ Similarly, Somali women living in Norway ${ }^{12}$ experience higher level of perinatal complications such as fetal distress and prolonged labour when compared with Norwegian-born women in the same setting.

Studies with migrant women from FGM practising countries in high income countries suggest that the poor birth outcomes could be related to cultural factors such as delays in seeking care, refusal of medical interventions and poor communication between women and care providers. ${ }^{12}$ Migrant women are often less able to understand treatment protocols or health information due to language issues, unfamiliarity with the health systems and a lack of shared decision making. ${ }^{13}$ 
Studies show that health providers in high-income countries are inadequately prepared to provide quality care to women who have undergone FGM as they are unfamiliar with the law in relation to FGM, or with clinical interventions that may be required, such as de-infibulation (a procedure to open up a closed vagina or type 3 FGM). ${ }^{14,15}$ Surgery (re-infibulation) after birth with the aim of re-suturing the vaginal opening $^{6}$ is illegal in many high income countries. Despite this, re-infibulation continues to occur due to lack of knowledge among providers and women. ${ }^{16-18}$

The needs and concerns of women with FGM receiving maternity care have not been fully investigated to inform the provision of care. This lack of knowledge makes it challenging for the health system and health care providers, to appropriately respond to women’s needs. ${ }^{19-21}$

We undertook a meta-synthesis of the qualitative evidence to explore the maternity care experiences and needs of migrant women with FGM in high-income countries. We sought to identify the gap between known aspects of quality maternity care and what migrant women who have undergone FGM perceive or experience as quality maternity care.

\section{Methods}

A meta-synthesis of qualitative studies was undertaken to provide an explanation of concepts and ideas across different high-income contexts to identify service gaps and provide evidence to support future maternity health service planning and models of care $^{22}$ for women with FGM.

Papers were included if they were peer reviewed, in English, published between 2000-2016 (to ensure relevancy of findings to current health systems), and focused on the maternity care experiences of women who have undergone FGM and migrated from high prevalence FGM countries ${ }^{5}$ to high-income countries. $^{23}$

An online search was conducted between June and August 2016 using the electronic databases PubMed, CINAHL, Medline, Scopus, Academic Search Complete, Science Direct, Web of Science, ProQuest 
Nursing and Allied Health Source, ProQuest Health and Medical Complete. In addition, hand and snowballing searches were undertaken involving an examination of the reference lists of articles reviewed to find other potentially relevant articles.

Original terms from the review questions and objectives were used to identify the search terms, as well using the MeSH terms. In addition, we explored a variety of combinations of potential terms identified within the relevant literature. (Table 1). In the initial search, 1265 papers were identified. After the removal of duplicates, 612 remaining papers' titles and abstracts were screened for relevance. Initially, 205 relevant studies were identified, and their full text was assessed for eligibility (Table 2).

In total, 22 papers were appraised using the critical appraisal skills program (CASP) systematic review checklist $^{24}$ (Supplementary table 1) Of the 22 articles appraised, six papers ${ }^{25-30}$ were excluded as they did not adequately address the quality criteria due to methodological limitations, relevance of results, or inadequate logical connection between results and conclusions. The three authors discussed and agreed upon the final selected papers (Figure1 and Table 3).

The analysis was undertaken by extracting all quotations from women participants from the 'Results' and 'Discussion' sections in each paper ${ }^{31}$. Data were imported into NVivo software to facilitate the thematic analysis by classification, coding and categorising of qualitative data as undertaken in previous meta-syntheses. ${ }^{32,33}$

We followed Thomas and Harden's ${ }^{33}$ recommended three steps for the thematic data synthesis: '1) Free line-by-line coding of the original quotations, 2) Grouping of similar concepts into descriptive themes, 3) Generating analytical themes that emerge from, and step beyond, the descriptive themes'.

The line-by-line coding focused on identifying the content and meaning of women's maternity care experiences. For example, sentences were coded as non-verbal disrespect if they included references such as 'dirty look', 'shocked’, ‘scared’, ‘surprised'. Patterns across the codes were then identified and codes grouped into categories. Finally, major themes and subthemes were discussed until consensus was 
reached among the authors. The synthesised ${ }^{34}$ data was presented in a visual 'mind map' (Figure 2) to provide deeper insight and a more coherent understanding of the health experiences and needs of women who have undergone FGM and migrated to a high-income country.

\section{Results}

Sixteen peer reviewed studies conducted in nine countries (four in United Kingdom, three in United States, two in Sweden, two in Australia and one each from Canada, Switzerland, Finland, France and Norway) were included. Most papers ( $n=11)$ focused on the experiences of Somali women and the remaining studies $(\mathrm{n}=5)$ had a mixed African study population including women from Ethiopia, Eritrea, Sudan, Kenya, Liberia and Somalia (Table 3). All studies were qualitative except for one descriptive survey $^{35}$ from which the qualitative data from the open questions were selected for this review.

Four themes were identified and are described below (Figure 2).

\section{Living with fear, stigma and anxiety}

Women's fear and anxiety was associated with their experiences of FGM, being in or encountering an unknown environment or unfamiliar situation, fear of death or fear of complications resulting from pregnancy and childbirth. ${ }^{16,17,35-46}$ Three analytical subthemes emerged including fear of complications, fear of caesarean section and feeling of shame and stigma associated with FGM.

The fear of caesarean section was influenced by peers, religion and culture. Their fear was irrespective of whether they had a previous caesarean section. Being afraid of a caesarean section was also influenced by information given by health providers. ${ }^{16,35,39,42}$ For instance, a woman said:

'...The doctor frightened me by saying you may not have a healthy or live baby as a result of your FC (female circumcision). I told him I believe in Allah who determines my baby's life ...I was very scared and afraid'. ${ }^{35}$ 
Sometimes fear of unexpected complications or interventions meant women avoided seeking health care. $^{35,36,41}$ Some described approaches to avoid a caesarean section such as limiting their diet to reduce fetal growth. ${ }^{47}$ This is illustrated by a Somali woman's quotation:

'Here in London, when you have long suffering (prolonged labour)... the baby is taken by caesarean. Therefore, I am afraid that if I arrive early and take a little time ... I will have to be operated .... It is better to wait ... Those who have given birth here have told me'. ${ }^{41}$

In some studies, women were afraid of a caesarean section as they believed it would take a long time for them to recover and would affect their ability to do daily housework, ${ }^{41,42}$ or their ability to become pregnant again. ${ }^{36,37}$

However, there were women with FGM who had undergone an elective caesarean section as they believed they might have a traumatic and complicated vaginal birth because they had previously experienced complications. ${ }^{43}$ For instance, one woman said:

'I thought because of the first experience, it was so horrible, I thought I could have a caesarean section, you know, I thought there's a chance of the tearing once again...I mean I kind of regret it. I wish I had a normal birth because I assumed a caesarean section is easier but only it's not easier. It's horrible!' 43

Feelings of shame, isolation, stigma and the loss of dignity were noted across studies, with stigma and shame being common emotions expressed by women. ${ }^{17,43,44}$ Women reported feeling different, unprotected and unsafe in the hands of health providers whom they perceived as strangers and incompetent. ${ }^{17,43,44}$ Feelings of shame and stigma sometimes hindered women's disclosure of FGM or any related complications they may have experienced, ${ }^{37,43}$ for example:

'Kept it [FGM] hidden because I thought it was kind of like embarrassing...so I didn't tell her, I didn't say I was scared'. ${ }^{43}$ 


\section{Feelings of vulnerability, distrust and discrimination}

Due to the social and emotional consequences of FGM, women felt vulnerable or helpless as they encountered the health system and health providers in a new country. They were traumatised by the negative judgments and racist comments they received, as well as disrespectful reactions by care providers. ${ }^{35,39,40,46}$ Women perceived that they experienced both verbal and non-verbal abuse, and discrimination ${ }^{16,35,37-40,44,45}$ for instance:

'They were very unkind and judgmental against my culture. They made me to feel unworthy to have a baby. They were sorry for my husband. One nurse said, 'a handsome young man to love a woman who cannot satisfy (him) sexually is a mystery to me'. ${ }^{35}$

Women were sometimes exposed to unnecessary and unjustified medical advices or interventions. Some were advised by the doctors to be sterilised or undergo a caesarean section, or seek health care from a health professional from their own community who was familiar with FGM $^{16,35,38,41,44,46,48}$ as the following quote illustrates:

'They did not understand that I could possibly have a vaginal delivery and the nurse told me that usually our doctors are not comfortable unless it is a caesarean section'.35

There was a lack of trust in providers' knowledge and skills relating to FGM. Women felt they had to guide health providers during childbirth because they believed their providers did not know how to manage FGM during pregnancy and childbirth and how and when to perform deinfibulation ${ }^{17,35,37,45,48}$ for instance:

'I told the midwife that I was circumcised and sutured. The midwife had to cut and open the stitches during my delivery. I was worried and feared how she was going to manage it'. ${ }^{17}$

\section{Dealing with past and present ways of life after resettlement}


Women were frustrated in their attempt to fit their own culture into the Western context. Many women described how cultural conflicts or differences presented them with difficulties in dealing with the host culture $^{17,35,37-40,43,44,46,48,49}$ for instance:

'I became a victim in Sudan already when I was 4 years old; I had no choice. Now I have to become a victim again after delivery, when the midwives refuse to re-suture me.... I understand that the law forbids them to do any suturing, but I am already injured; why do I have to suffer twice?' 37

Women talked about traumatic memories related to their experiences of undergoing FGM. ${ }^{37}$ Some also referred to being re-infibulated during maternity care in the host countries despite this being illegal. ${ }^{17,37}$ They compared their feelings of anguish and powerlessness when they underwent FGM to their experiences of health care in high-income countries ${ }^{17}$. For instance, a woman said:

'I was re-sutured twice. The second time was in Sweden. The midwife sewed without asking if I wanted or not. I was surprised, because I knew that it was illegal. After my third delivery, the midwife told me that the law prohibits re-infibulation, but the midwife stitched a little from each side as the opening was too wide'. ${ }^{17}$

The views of women indicated how deeply ingrained FGM is within their culture and personal identity. ${ }^{35,49}$ Some believed in the continuation of the practice ${ }^{46}$ and challenges to this confronted their own cultural norms. One woman said:

'We live in dangerous times. I do not think that circumcising the girls should be outlawed. They allow people to drink beer and alcohol, but they are saying circumcision is not good'. ${ }^{4}$

\section{Seeking support and involvement in health care}

Women described their feelings of incapability and hopelessness when they were not included in health care decision making processes and did not have a positive interaction with health care providers. 
Women felt a lack of control over their own bodies and their disengagement with health care resulted in further anxiety and stress. Communication and power relations were two analytical subthemes under this theme ${ }^{17,35-37,40,43,46,49}$ for example:

'They had tied this belt so tight around my belly, it pained me and I tried to pull it away. I tried to tell the nurse, but she didn't listen. She was angry and yelled at me that the heartbeat was indicating danger... So it ended like that, in a caesarean section. I still don't know whether it was necessary to cut me'. ${ }^{16}$

Women talked about their exclusion from health care decisions that were ultimately made by providers ${ }^{17}$ and sometimes by family members. ${ }^{43}$ At times, women were not asked if they had undergone FGM which contributed to their fear about how their birth would be managed. Some women seemed resigned to having decisions made on their behalf, even when the care providers' reasoning had not been communicated to them ${ }^{16,17,35,37,40}$ for example:

'I would have my appointments made for me and each time I went they would check or take what they wanted, and then I would leave without understanding what they had done. . . I saw no special kindness. They would just do the job and go' ${ }^{49}$

The language used by health care providers was often seen as scary or frightening. ${ }^{35}$ This resulted in misunderstandings between women and care providers which led to increased fear as shown in the following quote:

'I asked the doctor why I have to have a pelvic exam every time and with too many people and a long time on the table. He said 'because you are very risky'. This caused unnecessary stress. I could not sleep at night...I did not have anyone to talk to and I thought I had stomach cancer'. ${ }^{35}$ 
Just knowing the language was not always enough as women also reported making poor decisions about their treatment due to limited interpersonal communication or a lack of understanding about the information provided. ${ }^{35,45}$ Due to the sensitivity of the topic, interpreters did not always translate full conversations to health care providers. ${ }^{46}$ One women said

'I wish I had a way to communicate with the doctor directly. There are things that sometimes I want to say or ask but I feel embarrassed saying it through a translator, especially on the phone'. ${ }^{46}$

Women tended to prefer direct conversations with providers rather than receiving written information. ${ }^{45}$ Furthermore, they valued oral information given by midwives despite the fact they did not always understand it. ${ }^{49}$ Women were sometimes overwhelmed and confused with the amount of FGM-related information given to them at once. ${ }^{35,37}$

'You say, “Oh yes, yes.” Although you are not answering the questions, you just say yes ... "Are you alright?” "Yes.” "Did you understand?" "Yes.” "Yeah, and some of the women don't understand what the doctor said at all'. ${ }^{44}$

Despite the negative experiences of some women, they commonly expressed appreciation for the support they had received within the health system. ${ }^{17,35,38-40,44,48,49}$ They spoke positively about midwives who had knowledge of, and familiarity with, FGM as this made communication easier ${ }^{17,35,40}$ for example:

'I was lucky when I met a midwife in Sweden who knew about circumcised women. This was a great help to make me feel secure because it was my first time to be pregnant and to live far from my parents and family’. ${ }^{17}$

Women were happy when providers treated them like other women and did not focus on FGM as an abnormality or ask them details about their sexual experience. ${ }^{40,48}$ 
'...She didn't talk about it, and that didn't shock me. I didn't feel bad about it because I didn't hear the whisperings, there wasn't this movement of people passing by to look. She did her job ...; she behaved as if everything was normal'. ${ }^{40}$

\section{Discussion}

This qualitative meta-synthesis found that women's maternity care experiences in high-income countries were influenced by fear, stigma, vulnerability and unfamiliarity with a new environment and culture Additional underlying factors affecting women's experiences included perceived discrimination from health providers', limited FGM-related clinical knowledge and cultural competence. This review also identified women’s desire to be more involved in decisions concerning their care.

The findings of the review identify opportunities to improve the provision of quality maternity care. Quality care for this group of women should be multidimensional ${ }^{50,51}$, and go beyond medical care alone to address social and cultural issues. ${ }^{51}$ Quality maternity care should incorporate concepts such as woman-centredness, and cultural competence $e^{52,53}$ that are based upon an in-depth understanding of women's needs. ${ }^{50,54}$ One way that women's needs can be fully realised is by involving women themselves in the design, delivery and evaluation of health services. ${ }^{54}$

In our review, women with FGM who migrated to a high-income country were worried and frustrated by a mismatch between the information and resources they were given and those they wished to receive. All women should have access to timely and appropriate education and health information based on their needs. ${ }^{55}$ It is ideal if women themselves participate in a dynamic interaction where the information provided is consistently evaluated and modified according to their needs and the context of care. ${ }^{56,57}$ Many resources have been designed in high-income countries to guide health professionals during consultations with women with FGM. However, these materials are often generic and tend to focus on information provision as opposed to communication with women. ${ }^{58,59}$ Consistent with the findings of 
our meta-synthesis, women in other studies preferred concise information presented in plain language with the aid of visual communication tools such as short videos. ${ }^{60}$ Involving women in the design of such resources enables them to be contextually and culturally tailored to the specific needs of women in their host country. ${ }^{61}$

This meta-synthesis identified that all women despite their culture desired accessible, available and acceptable care. Where women received continuity of care from a culturally and clinically competent health care provider, they were more likely to trust the providers and share their cultural and health needs. ${ }^{62}$ A model of care specifically for women from vulnerable groups such as women affected by FGM should encompass continuity of care, educational interventions, and access to mental health support. ${ }^{63}$ Continuity of care enhances health care providers' knowledge and confidence and enables them to be more aware of women's socio-cultural needs which results in women feeling empowered and achieving a greater sense of control over their own care. ${ }^{64}$ Further, providing access to a continuum of specialised FGM services, along with standardised guidelines for health providers will potentially contribute to improved perinatal outcomes for women affected by FGM. ${ }^{10}$

Misconceptions concerning the provision of culturally sensitive health care affects the delivery of necessary interventions such as deinfibulation for women affected by FGM. For example, in Norway health care providers assumed that not cutting infibulated Somali women during pregnancy was culturally respectful, whereas Somali women feared not being opened before they gave birth. ${ }^{65}$ Despite the current policy discourse and global guidelines that promote a focus on respectful maternity care, cultural safety and clear communication, ${ }^{58,59,66,67}$ some health providers are either not aware of these guidelines or unable to put them into practice. ${ }^{14,68}$ Cultural competence in an enabling environment with sufficient strategies and policies in place could potentially make a positive impact upon health outcomes for women with FGM, and provide equitable access to services across ethnic groups. ${ }^{69,70}$ 
The findings of our review highlight the importance of shared decision making. In our review, language barriers and miscommunication were highlighted as challenges to shared decision-making. Evidence shows that choices concerning a woman's pregnancy may be made by their primary clinicians without full consultation with the woman. ${ }^{13}$ Whilst women usually consider birth a natural and empowering process, ${ }^{71,72}$ disregarding women's autonomy and involvement may disempower them, ${ }^{11,73,74}$ thereby preventing women from engaging in shared decision making. ${ }^{61}$

The findings of our synthesis provide insights from the perspective of affected women into issues concerned with the provision of their own care. Such insights are necessary to inform the provision of women-centred care, particularly for women from vulnerable groups whose voices are often unheard. However, a limitation of the review is that most included studies mostly focused on Somali women. Given the cultural diversity of women from different countries where FGM is prevalent, Somali women may report different experiences to women from other countries. In addition, women sometimes described their experiences of health services in general, rather than those specifically focused on maternity care. Finally, we only included articles written in English that met a limited inclusion criteria. As a result it is possible that some significant papers on FGM in other languages may have been omitted.

\section{Conclusions}

The findings suggest that future actions for improving maternity care quality should be focused on woman-centred practice, demonstrating cultural safety and developing mutual trust between a woman and her care providers. Meaningful consultations with women affected by FGM in high-income settings requires cultural sensitivity and acknowledgement of their specific circumstances. This can be achieved by engaging women affected by FGM in service design to provide quality care and ensure woman focused policy is developed and implemented. 


\section{References}

1. UN. Eliminating female genital mutilation: A Joint interagency statement. Geneva: UNAIDS, UNDP, UNECA, UNESCO, UNFPA, UNHCHR, UNHCR, UNICEF, UNIFEM, WHO.;2008.

2. Kaplan A, Cham B, Njie LA, Seixas A, Blanco S, Utzet M. Female genital mutilation/cutting: the secret world of women as seen by men. Obstetrics and gynecology international. 2013;2013:643780.

3. UNICEF. Female Genital Mutilation/Cutting: What might the future hold. New York: UNICEF;2014.

4. UNICEF. Female Genital Mutilation/Cutting: A statistical overview and exploration of the dynamics of change. New York: Unicef;2013.

5. UNICEF. Female Genital Mutilation/Cutting: A Global Concern. New York: UNICEF;2015.

6. WHO. WHO guidelines on the management of health complications from female genital mutilation. Geneva, Switzerland: World Health Organisation; 2016.

7. Dubourg D, Richard F, Leye E, Ndame S, Rommens T, Maes S. Estimating the number of women with female genital mutilation in Belgium. The European Journal Of Contraception \& Reproductive Health Care: The Official Journal Of The European Society Of Contraception. 2011;16(4):248-257.

8. Alison; M, Efua; D. Prevalence of Female Genital Mutilation in England and Wales: National and local estimates. UK: Department of Health's FGM Prevention Programme;2015.

9. Elnashar RA, Abdelhady R. The impact of female genital cutting on health of newly married women. International journal of gynaecology and obstetrics: the official organ of the International Federation of Gynaecology and Obstetrics. 2007;97.

10. Varol N, Dawson A, Turkmani S, et al. Obstetric outcomes for women with female genital mutilation at an Australian hospital, 2006-2012: a descriptive study. BMC pregnancy and childbirth. 2016;16:328.

11. Small R, Gagnon A, Gissler M, et al. Somali women and their pregnancy outcomes postmigration: data from six receiving countries. BJOG: International Journal of Obstetrics \& Gynaecology. 2008;115(13):1630-1640.

12. Vangen S, Stoltenberg C, Johansen REB, Sundby J, Stray-Pedersen B. Perinatal complications among ethnic Somalis in Norway. Acta obstetricia et gynecologica Scandinavica. 2002;81(4):317-322.

13. Origlia P, Jevitt C, Sayn-Wittgenstein Fz, Cignacco E. Experiences of Antenatal Care Among Women Who Are Socioeconomically Deprived in High-Income Industrialized Countries: An Integrative Review. Journal of midwifery \& women's health. 2017:n/a-n/a.

14. Leye E, Ysebaert I, Deblonde J, et al. Female genital mutilation: Knowledge, attitudes and practices of Flemish gynaecologists. European Journal of Contraception and Reproductive Health Care. 2008;13(2):182-190.

15. Dawson AJ, Turkmani S, Varol N, Nanayakkara S, Sullivan E, Homer CSE. Midwives' experiences of caring for women with female genital mutilation: Insights and ways forward for practice in Australia. Women and Birth. 2015;28(3):207-214.

16. Vangen S, Johansen REB, Sundby J, Træen B, Stray-Pedersen B. Qualitative study of perinatal care experiences among Somali women and local health care professionals in Norway. European Journal of Obstetrics \& Gynecology and Reproductive Biology. 2004;112(1):29-35.

17. Lundberg PC, Gerezgiher A. Experiences from pregnancy and childbirth related to female genital mutilation among Eritrean immigrant women in Sweden. Midwifery. 2008;24(2):214-225.

18. Moeed S, Grover S. Female genital mutilation/cutting (FGM/C): Survey of RANZCOG Fellows, Diplomates \& Trainees and FGM/C prevention and education program workers in Australia and New Zealand. Australian and New Zealand Journal of Obstetrics and Gynaecology. 2012;52:523-527.

19. Relph S, Inamdar R, Singh $\mathrm{H}$, Yoong W. Female genital mutilation/cutting: knowledge, attitude and training of health professionals in inner city London. European Journal of Obstetrics \& Gynecology and Reproductive Biology. 2013;168(2):195-198.

20. Hoang HT, Le $Q$, Kilpatrick S. Having a baby in the new land: a qualitative exploration of the experiences of Asian migrants in rural Tasmania, Australia. Rural and remote health. 2009;9(1):1084.

21. Ogunsiji O. Female Genital Mutilation (FGM): Australian Midwives' Knowledge and Attitudes. Health Care for Women International. 2015;36(11):1179-1193. 
22. Tong A, Flemming K, McInnes E, Oliver S, Craig J. Enhancing transparency in reporting the synthesis of qualitative research: ENTREQ. BMC Medical Research Methodology. 2012;12(1):181-188.

23. World Bank. High income: OECD. http://data.worldbank.org/income-level/OEC. Accessed April 2016, 2016.

24. CASP. Critical Appraisal Skills Programme . CASP Systematic Review Checklist. 2017; http://www.caspuk.net/checklists. Accessed Janury 2017.

25. Chalmers B, Hashi KO. 432 Somali Women's Birth Experiences in Canada after Earlier Female Genital Mutilation. Birth. 2000;27(4):227-234.

26. Andro A, Cambois E, Lesclingand $M$. Long-term consequences of female genital mutilation in a European context: Self perceived health of FGM women compared to non-FGM women. Social Science \& Medicine. 2014;106:177-184.

27. Connor JJ, Hunt S, Finsaas M, Ciesinski A, Ahmed A, Robinson BBE. Sexual Health Care, Sexual Behaviors and Functioning, and Female Genital Cutting: Perspectives From Somali Women Living in the United States. The Journal of Sex Research. 2016;53(3):346-359.

28. Gele AA, Kumar B, Hjelde KH, Sundby J. Attitudes toward female circumcision among Somali immigrants in Oslo: a qualitative study. International journal of women's health. 2012;4:7.

29. Norman K, Hemmings J, Hussein E, Otoo-Oyortey N. FGM is Always with Us: Experiences, Perceptions, Beliefs of Women Affected by Female Genital Mutilation in London: Results from a PEER Study. Research and Development, London. 2009.

30. Pavlish CL, Noor S, Brandt J. Somali immigrant women and the American health care system: Discordant beliefs, divergent expectations, and silent worries. Social Science \& Medicine. 2010;71(2):353-361.

31. Tong A, Jesudason S, Craig JC, Winkelmayer WC. Perspectives on pregnancy in women with chronic kidney disease: systematic review of qualitative studies. Nephrology Dialysis Transplantation. 2015;30(4):652-661.

32. Norhayati MN, Surianti S, Nik Hazlina NH. Metasynthesis: Experiences of Women with Severe Maternal Morbidity and Their Perception of the Quality of Health Care. PloS one. 2015;10(7):e0130452.

33. Thomas J, Harden A. Methods for the thematic synthesis of qualitative research in systematic reviews. BMC Medical Research Methodology. 2008;8:45-45.

34. Britten N, Campbell R, Pope C, Donovan J, Morgan M, Pill R. Using meta ethnography to synthesise qualitative research: a worked example. Journal of Health Services Research \& Policy. 2002;7(4):209215.

35. Chalmers BO-HK. What Somali women say about giving birth in Canada. Journal of Reproductive \& Infant Psychology. 2002;20(4):267-282.

36. Ameresekere M, Borg R, Frederick J, Vragovic O, Saia K, Raj A. Somali immigrant women's perceptions of cesarean delivery and patient-provider communication surrounding female circumcision and childbirth in the USA. International Journal of Gynecology \& Obstetrics. 2011;115(3):227-230.

37. Berggren V, Bergström S, Edberg A-K. Being Different and Vulnerable: Experiences of Immigrant African Women Who Have Been Circumcised and Sought Maternity Care in Sweden. Journal of Transcultural Nursing. 2006;17(1):50-57.

38. Carolan M, Cassar L. Antenatal care perceptions of pregnant African women attending maternity services in Melbourne, Australia. Midwifery. 2010;26(2):189-201.

39. Degni F, Suominen SB, El Ansari W, Vehviläinen-Julkunen K, Essen B. Reproductive and maternity health care services in Finland: perceptions and experiences of Somali-born immigrant women. Ethnicity \& Health. 2013;19(3):348-366.

40. d'Entremont M, Smythe L, McAra-Couper J. The sounds of silence-a hermeneutic interpretation of childbirth post excision. Health Care for Women International. 2014;35(3):300-319.

41. Essén B, Binder P, Johnsdotter S. An anthropological analysis of the perspectives of Somali women in the West and their obstetric care providers on caesarean birth. Journal of psychosomatic obstetrics and gynaecology. 2011;32(1):10-18.

42. Essen B, Johnsdotter S, Hovelius B, et al. Qualitative study of pregnancy and childbirth experiences in Somalian women resident in Sweden. BJOG : an international journal of obstetrics and gynaecology. 2000;107:1507 - 1512. 
43. Moxey JM, Jones LL. A qualitative study exploring how Somali women exposed to female genital mutilation experience and perceive antenatal and intrapartum care in England. BMJ open. 2016;6(1).

44. Murray L, Windsor C, Parker E, Tewfik O. The Experiences of African Women Giving Birth in Brisbane, Australia. Health Care for Women International. 2010;31(5):458-472.

45. Straus L, McEwen A, Hussein FM. Somali women's experience of childbirth in the UK: Perspectives from Somali health workers. Midwifery. 2009;25(2):181-186.

46. Upvall MJ, Mohammed K, Dodge PD. Perspectives of Somali Bantu refugee women living with circumcision in the United States: A focus group approach. International journal of nursing studies. 2009;46(3):360-368.

47. Essen B, Johnsdotter S, Hovelius B, et al. Qualitative study of pregnancy and childbirth experiences in Somalian women resident in Sweden. Br J Obstet Gynaecol. 2000;107:1507 - 1512.

48. Hill N, Hunt E, Hyrkäs K. Somali Immigrant Women's Health Care Experiences and Beliefs Regarding Pregnancy and Birth in the United States. Journal of Transcultural Nursing. 2012;23(1):72-81.

49. Bulman KH, McCourt C. Somali refugee women's experiences of maternity care in west London: a case study. Critical Public Health. 2002;12:365 - 380.

50. Renfrew MJ, McFadden A, Bastos MH, et al. Midwifery and quality care: findings from a new evidenceinformed framework for maternal and newborn care. The Lancet. 2014;384(9948):1129-1145.

51. Symon A, Pringle J, Cheyne $\mathrm{H}$, et al. Midwifery-led antenatal care models: mapping a systematic review to an evidence-based quality framework to identify key components and characteristics of care. $B M C$ pregnancy and childbirth. 2016;16(1):168.

52. Van den Broek N, Graham W. Quality of care for maternal and newborn health: the neglected agenda. BJOG: An International Journal of Obstetrics \& Gynaecology. 2009;116(s1):18-21.

53. Hulton LA, Matthews Z, Stones RW. Aframework for the evaluation of quality of care in maternity services. Highfield,Southampton: University of Southampton; 2000.

54. Berg M, Asta Ólafsdóttir Ó, Lundgren I. A midwifery model of woman-centred childbirth care - In Swedish and Icelandic settings. Sexual \& Reproductive Healthcare. 2012;3(2):79-87.

55. Davies MM, Bath PA. The maternity information concerns of Somali women in the United Kingdom. Journal of Advanced Nursing. 2001;36(2):237-245.

56. McKenzie PJ. Positioning Theory and the Negotiation of Information Needs in a Clinical Midwifery Setting. Journal of the American Society for Information Science \& Technology. 2004;55(8):685-694.

57. Levy V. Protective steering: a grounded theory study of the processes by which midwives facilitate informed choices during pregnancy. Journal of Advanced Nursing. 2006;53(1):114-122.

58. UK Gov. Multi-agency statutory guidance on female genital mutilation. In: Department for Education DoH, ed. UK: UK government; 2016.

59. Governent of Western Australia. Female Genital Cutting/Mutilation a guide for health professionals. In: Programs TWsHCS, ed. Western Australia2016.

60. CoHealth. Starting Conversations about Female Circumcision In: Cohealth, ed. Vol Standard License2015.

61. Green JM. Integrating Women's Views into Maternity Care Research and Practice. Birth: Issues in Perinatal Care. 2012;39(4):291-295.

62. Sandall J, Devane D, Soltani H, Hatem M, Gates S. Improving Quality and Safety in Maternity Care: The Contribution of Midwife-Led Care. The Journal of Midwifery \& Women's Health. 2010;55(3):255-261.

63. Correa-Velez I, Ryan J. Developing a best practice model of refugee maternity care. Women and Birth. 2012;25(1):13-22.

64. McCourt C, Pearce A. Does continuity of carer matter to women from minority ethnic groups? Midwifery. 2000;16(2):145-154.

65. Johansen RE. Care for infibulated women giving birth in Norway: an anthropological analysis of health workers' management of a medically and culturally unfamiliar issue. Med Anthropol Q. 2006;20(4):516544.

66. NSW Kids and Families Female Genital Mutilation/Cutting + Talking with Families + An Educational Resource. In: Health N, ed. NSW: NSW Health; 2014. 
67. WHO. WHO recommendations on antenatal care for a positive pregnancy experience. Geneva, Switzerland: World Health Organisation; 2016.

68. Turkmani S, Homer C, Varol N, Dawson A. A survey of Australian midwives' knowledge, experience, and training needs in relation to female genital mutilation. Women and Birth. 2017.

69. Beach MC, Price EG, Gary TL, et al. Cultural Competency: A Systematic Review of Health Care Provider Educational Interventions. Medical care. 2005;43(4):356-373.

70. Clarke M, Savage G, Smith V, et al. Improving the organisation of maternal health service delivery and optimising childbirth by increasing vaginal birth after caesarean section through enhanced womencentred care (OptiBIRTH trial): study protocol for a randomised controlled trial (ISRCTN10612254). Trials. 2015;16(542):1-9.

71. Gibbins J, Thomson AM. Women's expectations and experiences of childbirth. Midwifery. 2001;17(4):302-313.

72. Baker SR, Choi PY, Henshaw CA, Tree J. 'I Felt as though I'd been in Jail': Women's Experiences of Maternity Care during Labour, Delivery and the Immediate Postpartum. Feminism \& psychology. 2005;15(3):315-342.

73. Small R, Lumley J, Yelland J, Rice PL. Shared antenatal care fails to rate well with women of non-Englishspeaking backgrounds. The Medical journal of Australia. 1998;168(1):15-18.

74. Ali C, Strom A. "It is important to know that before, there was no lawalawa." Working to stop female genital mutilation in Tanzania. Reproductive health matters. 2012;20(40):69-75. 
Table 1: Keywords used in the electronic bibliographic database search

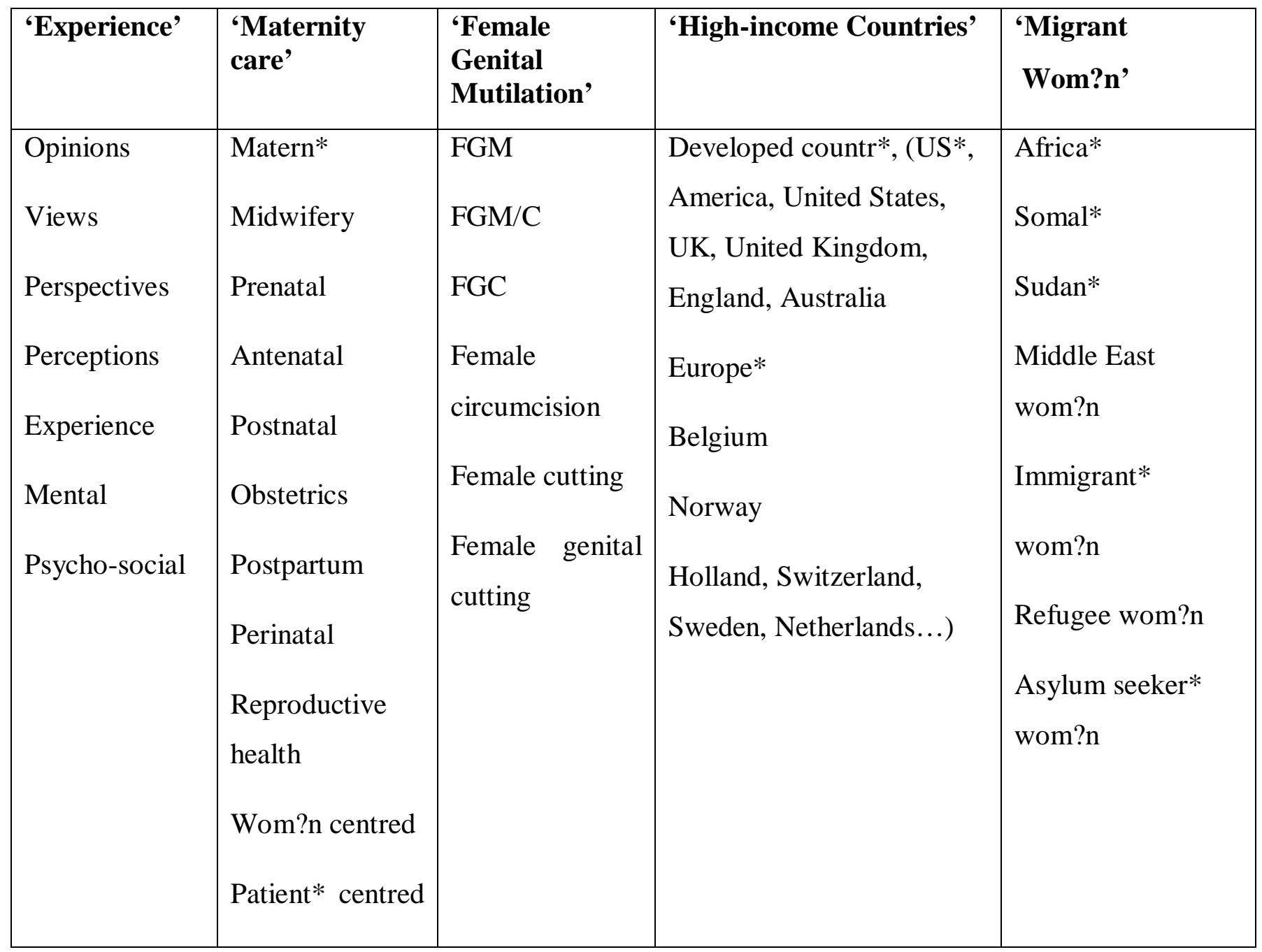


Table 2: Papers retrieved in the database Search

\begin{tabular}{|l|l|l|}
\hline Data Source & $\begin{array}{l}\text { Initial Search } \\
\text { Outcome }\end{array}$ & $\begin{array}{l}\text { Relevant } \\
\text { Papers }\end{array}$ \\
\hline Medline & 20 & 11 \\
\hline Pubmed & 24 & 13 \\
\hline Web of Science & 43 & 17 \\
\hline ProQuest Health and Medicine & 309 & 41 \\
\hline ProQuest Nursing and Allied Health & 110 & 25 \\
\hline Scopus & 316 & 35 \\
\hline Science Direct & 179 & 23 \\
\hline Academic Search Complete (EBSCO) & 57 & 21 \\
\hline CINAHL & 4 & 3 \\
\hline Hand searched & 203 & 16 \\
\hline Total & 1265 & 205 \\
\hline
\end{tabular}


Table 3: Characteristics of the Studies Included in the Meta-synthesis of maternity care experiences and needs of migrant women from female genital mutilation practising countries in high-income contexts

Author/

Context

Ameresekere

(2011)/

USA
Methodology

Qualitative-
semi structure interviews
Participants

23 Somali

women

Aim

\section{Major Finding}

To explore perceptions of
caesarean delivery and pat
provider communication

Women feared postoperative complications that may result from caesarean delivery and their effect on daily life and the reactions of peers. Women felt that labour was rushed by physicians. Communication with providers was reported to be poor and providers infrequently discussed FGM, or explained its effect on childbirth experiences.

$\begin{array}{ll}\text { Berggren } & \text { Qualitative- } \\ \text { (2006)/ } & \text { in-depth } \\ \text { Sweden } & \text { interviews }\end{array}$

\section{2 women \\ from \\ Somalia, \\ Sudan, \& \\ Eritrea.}

$\begin{array}{ll}\text { Carolan } & \text { Qualitative } \\ \text { (2010)/ } & \text { in-depth } \\ \text { Australia } & \text { interviews }\end{array}$

Chalmers (2002)/

Canada

\begin{tabular}{|c|c|}
\hline $\begin{array}{l}18 \text { pregnant } \\
\text { women } \\
\text { (Sudan, } \\
\text { Ethiopia, } \\
\text { Eritrea, } \\
\text { Somalia, } \\
\text { Kenya) }\end{array}$ & $\begin{array}{l}\text { To explore the experiences and } \\
\text { concerns of an African-born } \\
\text { sample of pregnant women } \\
\text { receiving antenatal care. }\end{array}$ \\
\hline $\begin{array}{l}432 \text { Somali } \\
\text { women }\end{array}$ & $\begin{array}{l}\text { To explore recent perinatal } \\
\text { experiences of Somali women } \\
\text { in Canada. }\end{array}$ \\
\hline
\end{tabular}

To explore women's experiences of encounters with Swedish maternal health care.

Women with FGM reported feeling different and vulnerable. They felt that they were looked down on by health care personnel because they had undergone FGM.
African women undergo a process of adjustment as they travel from a view of pregnancy in their country of origin as not 'special' to valuing continuous antenatal care and other supportive services offered to them in Australia.

Women perceived their maternity care to be disrespectful, harsh and offensive to their cultural values. Women considered their care to be inappropriate and rarely discussed birth practices and care management with their providers. 


$\begin{array}{lll}\text { Degni } & \text { Qualitative } & 70 \text { Somali } \\ \text { (2013)/ } & \text { focus group } & \text { women } \\ \text { Finland } & \text { discussions } & \end{array}$

d'Entremon

(2014)/

France

Qualitative (hermeneutic phenomenolog ical) interviews

$\begin{array}{ll}\text { Essén } & \text { Qualitative } \\ \text { (2000)/ } & \text { in-depth } \\ \text { Sweden } & \text { interviews }\end{array}$

\section{Essén}

(2011)/

England

Bulman

(2002)/

UK
Qualitative

(naturalistic inquiry)

in-depth interviews \&

focus group

discussions

Qualitative descriptive semistructured interviews \&

\section{4 women \\ (country not \\ identified)}

To explore immigrant Somali women's experiences and perceptions about reproductive and maternity health care services and service providers' cultural and communication competencies.

To understand the maternity needs of women with FGM.

15 Somali
women

To understand how cultural factors affect perinatal outcomes and explore women's pregnancy related attitudes and behaviours.

39 Somali women

12 Somali women
To explore the attitudes, perceptions and experiences of women in relation to caesarean delivery and address the relationship between Somali women and their western obstetric care providers.

To understand the views and experiences of Somali women in their contacts with the maternity services in the UK.
Participants were satisfied with the reproductive and maternity health care services they received. However, they perceived health care providers' communication to be poor and their attitudes unfriendly.

Despite receiving regular and early antenatal care, women reported that FGM was not discussed. Women emphasized the need for midwives to communicate with them, to break the taboo of silence, and to talk openly about FGM in a respectful manner.

Fear of death and caesarean section forced many women to decrease their food intake to diminish the growth of the foetus, thereby avoiding caesarean section and mortality.

Women believed there to be a strong association between caesarean birth and maternal death. Therefore they make what they perceive to be rational choices to avoid caesarean birth.

Poor management of FGM during childbirth, and communication and language difficulties were found to be the most important issues for women. These had negative implications for all aspects of their care. 


\begin{tabular}{|c|c|c|}
\hline $\begin{array}{l}\text { Hill } \\
\text { (2012)/ } \\
\text { USA }\end{array}$ & $\begin{array}{l}\text { Qualitative } \\
\text { (phenomenolo } \\
\text { gy) } \\
\text { focus group } \\
\text { discussions }\end{array}$ & $\begin{array}{l}18 \text { Somali } \\
\text { women }\end{array}$ \\
\hline $\begin{array}{l}\text { Lundberg } \\
\text { (2008)/ } \\
\text { Sweden }\end{array}$ & $\begin{array}{l}\text { Qualitative } \\
\text { (ethnographic } \\
\text { approach) } \\
\text { semi- } \\
\text { structured } \\
\text { interviews }\end{array}$ & $\begin{array}{l}15 \text { Eritrean } \\
\text { women }\end{array}$ \\
\hline $\begin{array}{l}\text { Moxey } \\
(2016) / \\
\text { UK }\end{array}$ & $\begin{array}{l}\text { Qualitative } \\
\text { (exploratory) } \\
\text { in-depth } \\
\text { interviews }\end{array}$ & $\begin{array}{l}10 \text { Somali } \\
\text { women }\end{array}$ \\
\hline $\begin{array}{l}\text { Murray } \\
\text { (2010)/ } \\
\text { Australia }\end{array}$ & $\begin{array}{l}\text { Qualitative } \\
\text { (phenomenolo } \\
\text { gy) } \\
\text { semi structured } \\
\text { interviews }\end{array}$ & $\begin{array}{l}10 \text { African } \\
\text { refugees (2 } \\
\text { Sudanese, } \\
2 \text { Ethiopian, } \\
2 \text { Somali, } \\
1 \text { Liberian) }\end{array}$ \\
\hline
\end{tabular}

To explore women's health care experiences and beliefs regarding pregnancy and birth.

To explore women's experiences of FGM during pregnancy, childbirth and the postpartum period.

\section{To explore women's} experience and perceptions of antenatal and intrapartum care, de-infibulation, caesarean section and vaginal delivery and factors that affect access to these services.

To explore the birth

experiences of African refugee women in Brisbane/Australia.

1 Liberian)
Women were faced with new experiences and information. They interpreted, and reacted to this information by integrating it within their own cultural, religious, and scientific beliefs.

Most women experienced complications during pregnancy, childbirth and in the postpartum period. Women stated that knowledgeable and well trained providers would improve the management of FGM during childbirth and reduce their risk of complications.

Women delayed de-infibulation until labour to avoid undergoing multiple operations if an episiotomy was anticipated. They reported that midwives were aware of FGM that led to open communication and stronger relationships, resulting in more positive experiences.

Participants experienced an overwhelming sense of fear loneliness and unfamiliarity as often they had no prior contact with the Australian health system.

Women often were surprised that midwives did not appear experienced in managing FGM were frustrated with lack of continuity of care. 

approach)

\begin{tabular}{|c|c|c|}
\hline $\begin{array}{l}\text { Upvall } \\
\text { (2009)/ } \\
\text { USA }\end{array}$ & $\begin{array}{l}\text { Qualitative- } \\
\text { focus group } \\
\text { discussions }\end{array}$ & $\begin{array}{l}23 \text { Somali } \\
\text { women }\end{array}$ \\
\hline $\begin{array}{l}\text { Vangen } \\
\text { (2004)/ } \\
\text { Norway }\end{array}$ & $\begin{array}{l}\text { Qualitative } \\
\text { in-depth } \\
\text { interviews }\end{array}$ & $\begin{array}{l}23 \text { Somali } \\
\text { women }\end{array}$ \\
\hline
\end{tabular}

To explore perceptions and experiences of childbirth.

To explore health care experiences.

To explore how perinatal care practice may influence labour outcomes among circumcised women.
Existing pressures as a consequence of migration were compounded by Somali women's experiences of childbirth. Inadequate care was reportedly provided during pregnancy and labour. The importance of Somalia's oral culture was not recognised when addressing communication barriers, Women reported a lack of continuity of care and felt that midwives held stereotyped and negative attitudes towards them.

Circumcision is considered a normal part of everyday life. Communication and language barriers were considered obstacles to receiving care as they limit women's ability to ask health related questions.

Somali women felt disempowered. Women feared caesarean birth and lack of providers' knowledge on FGM. 


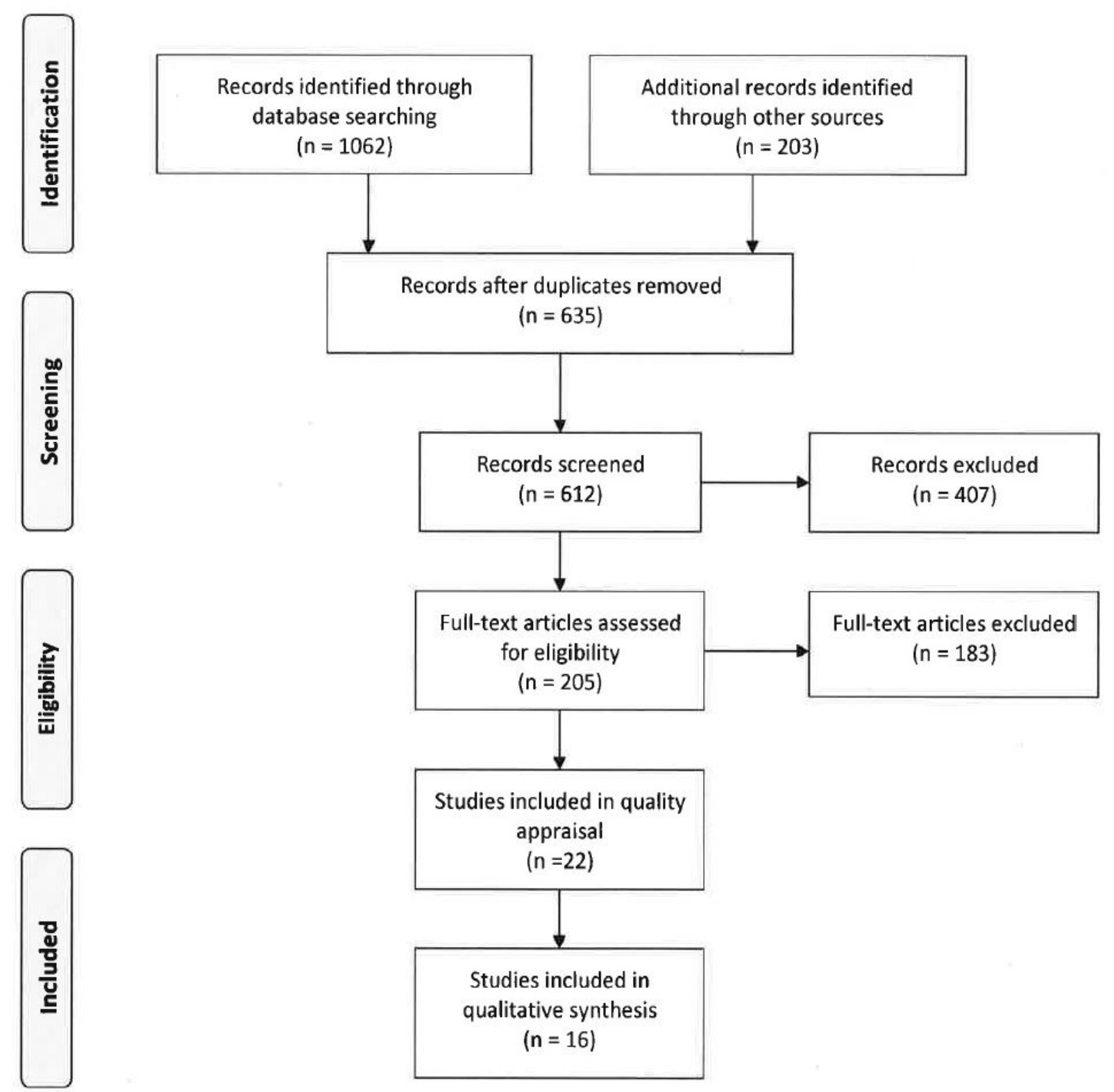

1

2 Figure 1: Search and Study Selection 


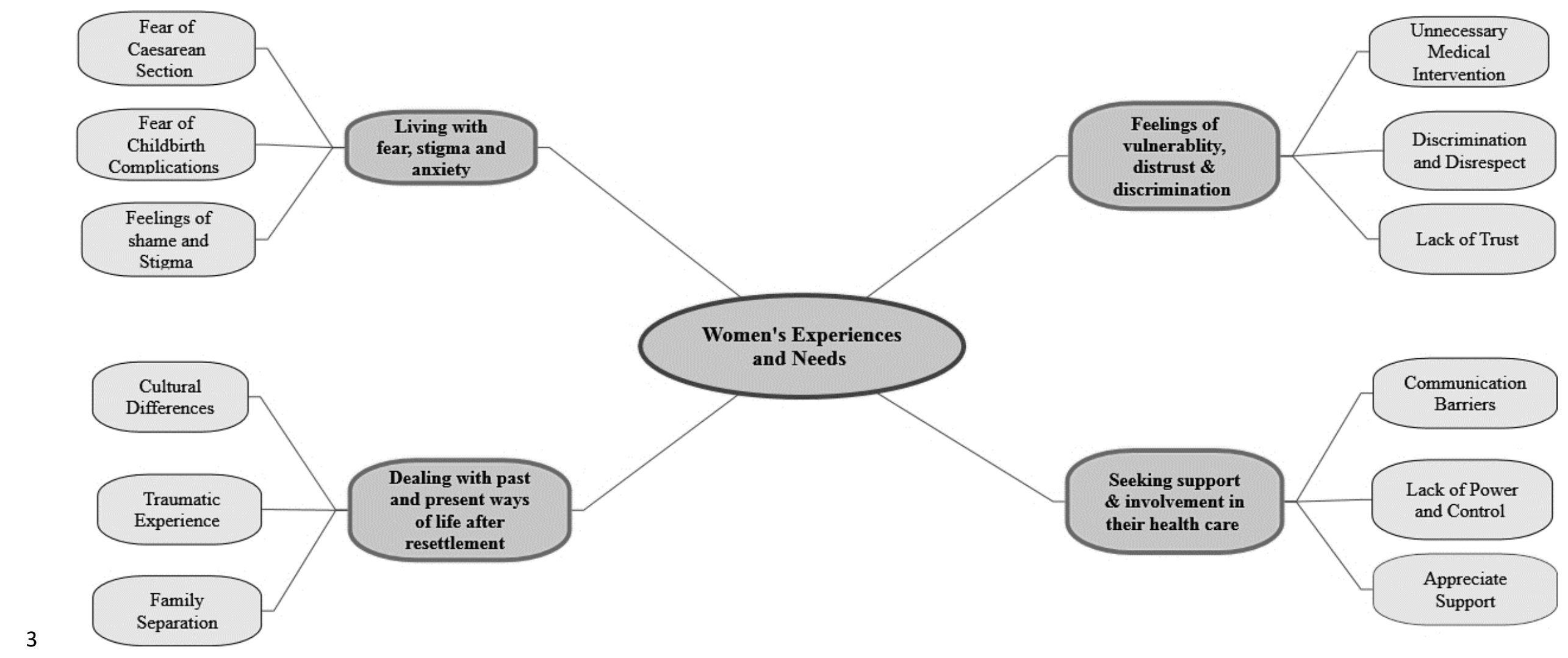

$4 \quad$ Figure 2: Themes derived from the analysis of the findings sections of the 16 included studies in the Meta-synthesis 